\title{
Improving executive function for better diabetes management
}

\begin{abstract}
Type 2 diabetes (T2D) is a global healthcare epidemic. In addition to physical consequences of the disease, T2D patients also experience cognitive decline. Importantly, intact cognitive function - and in particular, executive functioning - is essential for management of the disease. One way to improve executive functions is through exercise; indeed, there is a growing body of research that suggests that both aerobic- and resistance-training can improve aspects of executive function, both behaviorally and through changes in functional activation in the brain. Thus, we contend that improving executive function through strategic exercise interventions has the potential to both directly and indirectly impact the management and progression of the disease in T2D patients. Future directions and recommendations are discussed.
\end{abstract}

Keywords: exercise, type 2 diabetes, T2D, cognitive function, executive function
Volume 7 Issue 5 - 2017

\author{
Lindsay S Nagamatsu,' Joyla A Furlano² \\ 'School of Kinesiology, Western University, Canada \\ ${ }^{2}$ Department of Neuroscience, Western University, Canada
}

Correspondence: Lindsay S Nagamatsu, School of Kinesiology, Western University, 3M Centre, Room 2225, London, ON, Canada, Tel 519661211182659, Fax 5196613092,

Email lindsay.nagamatsu@uwo.ca

Received: December 13, 2017 | Published: December 2I, 2017

\section{Commentary}

Diabetes has become an epidemic in the United States, with over 30 million people currently affected, the majority of which have type 2 diabetes (T2D). ${ }^{1}$ At the societal level, it is the seventh leading cause of death in the US, and the burden on the health care system is estimated at $\$ 245$ billion in direct and indirect costs. ${ }^{1}$ Secondary consequences of T2D include microvascular and macrovascular complications, including neuropathy, coronary artery disease, and stroke. However, it is now widely recognized that patients with T2D also experience reduced cognitive function and are at greater risk for dementia and Alzheimer's disease. ${ }^{2}$ For example, in a large prospective study of more than 9,000 older women, those with diabetes had significantly worse cognitive performance at baseline and at six-year follow-up compared to their non-diabetic counterparts. ${ }^{3}$ Furthermore, dementia and T2D often co-occur, with up to $81 \%$ of older adults with dementia also having diabetes or impaired fasting glucose. ${ }^{4}$ Cognitive decline in T2D patients is a major concern because it can directly impact the management of the disease. Specifically, lifestyle modifications (e.g., diet and exercise) and self-management (e.g., monitoring of glucose levels and medication use) serve as the foundation for controlling the symptoms and trajectory of T2D. However, effective adoption and adherence to such strategies relies on executive functions - our higher level goal-directed cognitive processes, such as planning, conflict monitoring, and inhibition; indeed, previous research has linked executive functioning to engagement in health behaviours. Specifically, performance on the Stroop task significantly predicted smoking, substance abuse, and sleep habits in a sample of 217 adults. Further evidence comes from a cross-sectional study of 1,398 older adults with diabetes where those with greater cognitive impairment had lower adherence to both diet and exercise. ${ }^{6}$ Thus, improving executive functioning in those with T2D may positively impact management and progression of the disease. As evidence, in a ten year prospective study, performance on a test of executive functioning predicted survival time in those diagnosed with a chronic illness, including T2D.?

One theory explaining this connection between executive functioning and health behaviours is temporal self-regulation theory, which proposes that regulating our health behaviours requires the engagement of neurocognitive processes - and more specifically, executive functions. ${ }^{8}$ In particular, we must inhibit our temptation to give in to short term rewards, focusing instead on the potential longterm benefits. ${ }^{8}$ A common example is the decision to go to the gym, compromising the short-term consequences of the time commitment and uncomfortable feeling of exercising, with the long-term goal of improving future fitness and health. Success in this scenario requires our executive functions to inhibit, plan, and make complex decisions.

Based on the above, it would stand to reason that improving executive functioning would lead to better self-regulation, and therefore increase adherence to behaviours necessary to manage one's current disease state. One proposed method for improving executive functioning is through exercise. There is a growing body of literature to support the idea that both aerobic (AT) and resistance (RT) training can improve behavioural measures of executive functioning, as well as facilitate functional and structural plasticity in the brain. For example, Colcombe and colleagues ${ }^{9}$ found that healthy older adults who engaged in six months of thrice-weekly walking improved their performance on the flanker task, a test of response inhibition, and showed increased activation in frontal and parietal brain regions and decreased activation in the anterior cingulate cortex - exactly the regions of the brain required to regulate our health behaviours. ${ }^{8}$ These benefits to cognition from exercise have also been demonstrated in those with glucose intolerance, where older adults randomized to a six-month aerobic exercise group significantly improved their executive function compared to controls. ${ }^{10}$

While the majority of research on the effects of exercise on cognition have focused on AT, RT has also shown positive results. For example, twice weekly progressive resistance training has been shown to improve behavioural measures of executive function and result in functional brain plasticity in both healthy older women ${ }^{11,12}$ and those with mild cognitive impairment. ${ }^{13,14}$ Importantly, RT may provide even greater benefits to those with diabetes. In particular, one mechanism through which RT has been hypothesized to exert its impact on neural plasticity is through increasing levels of insulin-like growth factor 1 (IGF-1). ${ }^{15}$ Low levels of IGF-1 have been associated with increased risk of T2D, ${ }^{16}$ and may contribute to the cognitive deficits observed in this population. Because RT has additional benefits (e.g., improved 
skeletal muscle and bone health) in T2D patients ${ }^{17}$ and provides them with an alternative form of exercise which may increase uptake and adherence, we propose that future studies should examine the effects of RT on cognition in T2D patients. We contend that exercise is a promising strategy to not only mitigate the physical risk factors associated with T2D, but also the cognitive risk factors, which may in turn, improve physical factors through adherence to health strategies. Questions that remain to be answered include:

a. What are the specific cognitive functions that are impaired in those with diabetes?

b. What are the underlying mechanisms for the relationship between impaired cognition and T2D?

c. What type of exercise has the biggest impact for improving executive function in those with T2D?

Based on the above, the potential for exercise to improve T2D outcomes both directly and indirectly is clear. Notably, exercise is a promising, cost-effective, and scalable solution to improve executive function in those with T2D, and may result in increased quality of life and longevity in this high-risk patient population.

Thus, we conclude that large-scale high quality randomized controlled trials (RCTs) with executive function as the primary outcome are necessary to elucidate the potential benefits of exercise on the brain as well as the body in T2D patients.

\section{Acknowledgements}

This work was supported by funds awarded from Western's Strategic Support for CIHR Success program.

\section{Conflict of interest}

The author declares no conflict of interest.

\section{References}

1. Center of Disease Control and Prevention. National Diabetes Statistics Report, 2017. USA: US Department of Health and Human Services; 2017.

2. Awad N, Gagnon M, Messier C. The relationship between impaired glucose tolerance, type 2 diabetes, and cognitive function. J Clin Exp Neuropsychol. 2004;26(8):1044-1080.

3. Gregg EW, Yaffe K, Cauley JA, et al. Is diabetes associated with cognitive impairment and cognitive decline among older women? Study of Osteoporotic Fractures Research Group. Arch Intern Med. 2000;160(2):174-180.
4. Janson J, Laedtke T, Parisi JE, et al. Increased risk of type 2 diabetes in Alzheimer disease. Diabetes. 2004;53(2):474-481.

5. Hall PA, Elias LJ, Crossley M. Neurocognitive influences on health behavior in a community sample. Health Psychol. 2006;25(6):778-782.

6. Feil DG, Zhu CW, Sultzer DL. The relationship between cognitive impairment and diabetes self-management in a population-based community sample of older adults with Type 2 diabetes. J Behav Med. 2012;35(2):190-199.

7. Hall PA, Crossley M, D Arcy C. Executive function and survival in the context of chronic illness. Ann Behav Med. 2010;39(2):119-127.

8. Hall PA, Fong GT. Temporal self-regulation theory: A model for individual health behavior. Health Psychol Rev. 2007;1(1):6-52.

9. Colcombe SJ, Kramer AF, Erickson KI, et al. Cardiovascular fitness, cortical plasticity, and aging. Proc Natl Acad Sci USA. 2004;101(9):33163321.

10. Baker LD, Frank LL, Foster-Schubert K, et al. Aerobic exercise improves cognition for older adults with glucose intolerance, a risk factor for Alzheimer's disease. J Alzheimers Dis. 2010;22(2):569-579.

11. Colcombe SJ, Kramer AF, Erickson KI, et al. Resistance training and executive functions: A 12-month randomized controlled trial. Arch Intern Med. 2010;170(2):170-178.

12. Liu Ambrose T, Nagamatsu LS, Graf P, et al. Resistance training and functional plasticity of the aging brain: A 12-month randomized controlled trial. Neurobiol Aging. 2012;33(8):1690-1698.

13. Lindsay S Nagamatsu, Alison Chan, Jennifer C Davis, et al. Physical activity improves verbal and spatial memory in older adults with probable mild cognitive impairment: A 6-month randomized controlled trial. J Aging Res. 2013;2013:1-10.

14. Nagamatsu LS, Handy TC, Hsu CL, et al. Resistance training promotes cognitive and functional brain plasticity in seniors with probable mild cognitive impairment. Arch Intern Med. 2012;172(8):666-668.

15. Cassilhas RC, Lee KS, Fernandes J, et al. Spatial memory is improved by aerobic and resistance exercise through divergent molecular mechanisms. Neuroscience. 2012;202:309-317.

16. Huffman J, Hoffmann C, Taylor GT. Integrating insulin-like growth factor 1 and sex hormones into neuroprotection: Implications for diabetes. World J Diabetes. 2017;8(2):45-55.

17. Wood RJ, O’Neill EC. Resistance Training in Type II Diabetes Mellitus: Impact on areas of metabolic dysfunction in skeletal muscle and potential impact on bone. J Neutr Metab. 2012;268197:13. 